\title{
Design and Evaluation New Rice Planter Tool without Mechanical Engine for Improving Conventional Farmer's Posture as Revival of Agriculture's Indonesia
}

\author{
Amarria Dila Sari ${ }^{1, a}$, Nurmala Pusfitasari ${ }^{2, b}$ and Riza Rahma ${ }^{3, c}$ \\ ${ }^{1}$ Department of Industrial Engineering Universitas Islam Indonesia, Yogyakarta, Indonesia \\ ${ }^{2}$ Department of Industrial Engineering Universitas Islam Indonesia, Yogyakarta, Indonesia \\ ${ }^{3}$ Department of International Program of Industrial Engineering Universitas Islam Indonesia, \\ Yogyakarta, Indonesia \\ amarria.dila@gmail.com,nurmala.pusfita@gmail.com,rizarahmapanduwiranita@yahoo.com
}

Keywords: Participatory Ergonomic, Musculoskeletal Disorders, REBA, Rice Planter Tool Design

\begin{abstract}
Indonesia is an agricultural country and most of the population work in that sector. Indonesia focusses its development on the agricultural sector which emphasize on rice plantation. One of the obstacle on the agricultural sector in Indonesia is the conventional work method that is manually planting the rice in the rice field. However, this kind of method can affect the musculoskeletal system. Musculoskeletal discomfort complained because of excessive muscle contraction as an effect of heavy workload in a long duration. The objectives of this research was designing rice planter tool using participatory ergonomic approachment. This design could reduce the injury risks and musculoskeletal complaints and increase the productivity. There was a comparison between the proposed rice planter tool design with the conventional method of rice planting based on the musculoskeletal complaints, time efficiency and expended cost. TheREBA scoring result indicates that new-concept of rice planter tool improves subjective comfort level and reduces injury risks in the trunk, waist, right and left shoulders. By using the rice planter tool, the injury risk can be reduced until $66.67 \%$. On the other side, suppose the same worker which usually plants one rice sprout with conventional method spends time about x seconds. Compared by using the proposed rice planter tool, the worker only spends $0,5 \mathrm{x}$ seconds from conventional method rice planting. Therefore, the time for rice planting will be more efficient by using this tool.
\end{abstract}

\section{Introduction}

Musculoskeletal disorders (MSDs) are common health problem throughout the world. Assessment of exposure levels to MSDs risk factors can be an appropriate base for planning and implementing interventional ergonomics programs in the workplace. In many rapidly developing countries, where labor is cheap, proper posture analysis methods are often neglected. Proper posture arrangements and work environment are seldom provided. The workers, being economic migrants, accept adverse conditions as a part of the job and mostly work in bad posture. Repetitive processes and manual material handling are the major problems in the hand tool manufacturing. Therefore, musculoskeletal disorders (MSDs) are related to such high repetitive processes and working in bad posture. Thus, to improve the efficiency of the workers their posture needed tobe assessed and corrective measures should be adopted to avoid the musculoskeletal disorders[1].

Many parties have become more consider about MSDs' because of the emerged impact. It is shown from the result of previous research about MSDs'. An overview of previous research identifying: 1) the prevalence, types, and causes of farm-related injuries, paying particular attention to musculoskeletal disorders and the special populations within the agricultural sector, 2) interventions that have been developed to reduce risk factors associated with farm-related injuries, and 3) major initiatives which have been established to address health and safety issues in the agricultural sector[2]. 
Indonesia is an agricultural country that focuses its development on the agricultural sector which emphasize on rice planting. Agricultural sector, especially rice planting activity, the repetitive motion and work posture of the farmer can lead to MSDs' risks. Based on Nordic Body Map Questionnaire (NBMQ) in the preliminary study in Bantul, there are 4 body parts that have high illness value which are back, waist, right and left shoulders. While from the measurement result of Rapid Entire Body Assessment (REBA), final score shows the value of 10 which means that the activity has very high risk level and therefore the corrective actionis necessarry to be done now.

In order to reduce MSDs' risk, participatory Ergonomics' approach of Indonesian culture was considered in the proposed design of rice planter tool. The design also accommodated the conventional characteristics of Indonesian farmer, where most of the population is women. In addition, the farmer's productivity had also been increased. There were comparisons between rice planting activity by using proposed design of rice planter tool and conventional method in terms of musculoskeletal complaints, time efficiency and expended cost.

\section{Method}

\section{Research Subject}

The observation was conducted on 12 rice planting workers in Bantul, Indonesia with gender either male or female around 35 - 50 years old and Javanese ethnical. The time that needed to finish 1 rice field is about $3-4$ hours. Commonly, the conventional method of rice planting activity is done in groups.

\section{Research Procedure}

The overall research procedure is described above. There are several procedures that should be done for this research:

- Participatory Ergonomic: It is combination of organizational design to solve the ergonomic problem. All of elements in organization structure are gathered in a forum to discuss about ergonomic issue [3]. There were three steps of participatory ergonomic, were: participants selection, design and development, and implementation [4]. Participant's selection was done by the observer, and then the group was made. The group consists of the farmers, tool designer, ergonomic expert, and tool technician. The group did participatory activities in accordance with each job description (farmers: deliver the MSDs problem, tool designer: designs the tool based on the farmer's problem, ergonomic expert: gives recommendation relates with ergonomic view in product designing, and tool technician: gives recommendation relates with the tool technique such as material and price of the tool. The ergonomic expert does the interview directly in field where the farmers are planting the rice plants. The questions are about how to plant the rice and the culture of rice planting in the region. By using interview, the famer gives the ergonomic expert recommendation how to develop the product.

- Nordic Body Map Questionnaire: Working with awkward posture can cause musculoskeletal disorders [5]. The questionnaire distributions are conducted to know the farmers' complaints when planting rice plants conventionally or using the proposed auxiliary apparatus. Based on the of weight multiplication in questionnaires, the obtained result will be translated into bar chart then the body parts that have high musculoskeletal complaints will be known. It will become consideration in making the design of rice planter tool in order to reduce the complaints level.

- Working Time: The data collection is in form of working time when planting rice plants conventionally or using the proposed auxiliary apparatus.

- Work Posture Data: The data of work postures are collected by taking pictures of the farmers while planting rice plants. The data processing of work postures are done using REBA method. From the data processing, it will result the scores, risk levels and the corrective action that should be done. 
- Anthropometry Data: The anthropometry data that are taken about 17 body dimension of farmers that will be used as the measurement of the proposed design of rice planting tool. The dataprocessing are done by calculating the sufficiency, uniformity, normality and percentile data so that it will be obtained the measure of 17 body dimension of respondents.

- Work Method Comparison: In this phase, there will be a comparison between the conventional method and the using of rice planter tool in terms of musculoskeletal complaints, time efficiency and expended cost.

\section{Results}

\section{Nordic Body Map Questionnaire}

Based on the processing result of Nordic Body Map Questionnaire (NBMQ), it is obtained that there are strain and complaintin the waist of the farmer while planting the rice plant with score 34 , the score in right shoulder is 30, left shoulder is 29 and back score is 29 (Fig.1).

\section{Recapitulation of Nordic Body Map Questionnaire}

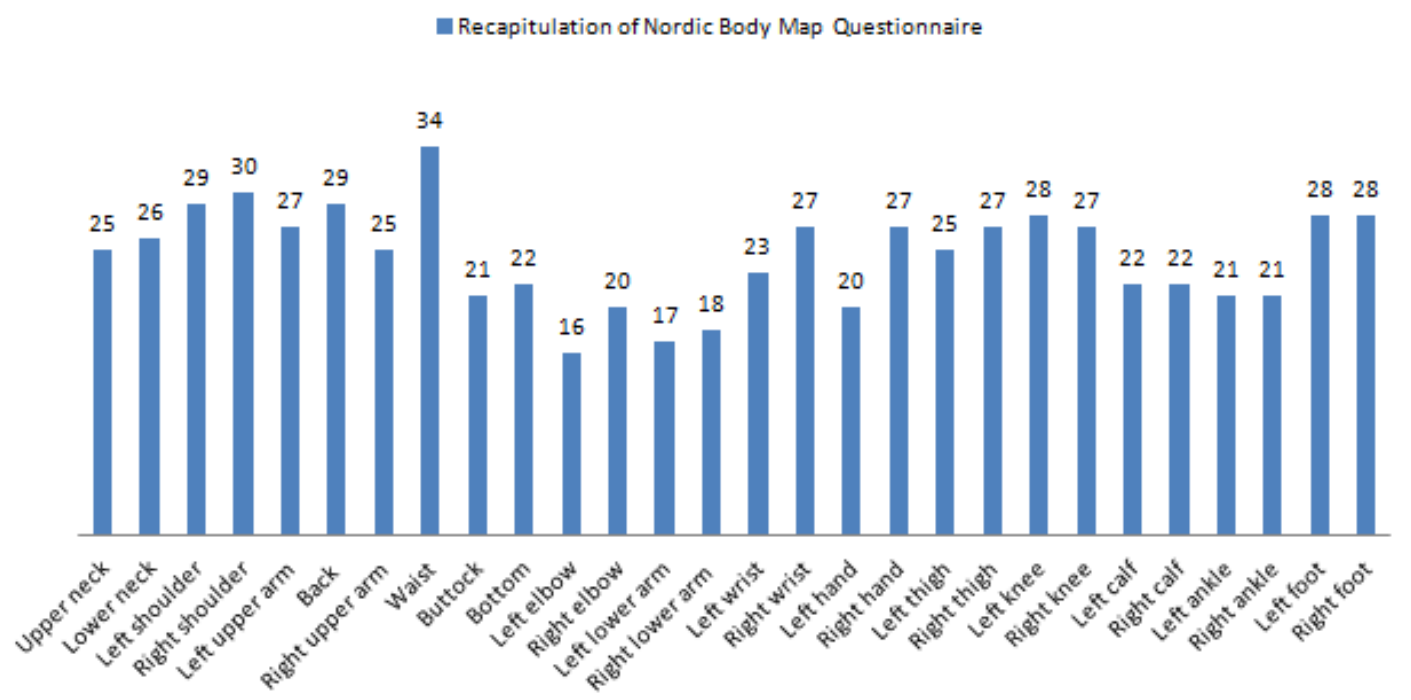

Fig. 1 The graph of NBMQ recapitulation when farmers conducting rice planting activity

\section{Work Posture using REBA Method}

The lines of each visible body segments are drawn according to the work posture and used as an input of REBA processing data (Fig.2). It can be seen the angle formed towards $\mathrm{x}$ - axis,on the neck is $32.5^{\circ}$, upper arm is $18^{\circ}$, lower arm is $26^{\circ}$, wrist is $38^{\circ}$, trunk is $111^{\circ}$ and on leg is $21^{\circ}$ (Fig.2)

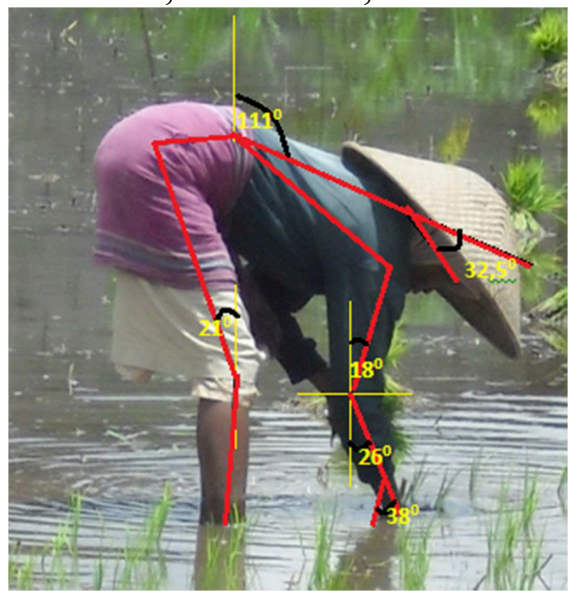

Fig.2Work posture of a farmer when planting rice 
REBA worksheet is used to assess the work position or neck posture, trunk, arm and leg of a worker rapidly. Besides that, this method also influenced by coupling factor, external load that supported by the body and the worker's activities. The processing data based on the angle formed, the score for table $\mathrm{A}$ is 7 with the score for neck is 3 , score for trunk is 5 , score for leg is 1 , and the load value is 0 (load $<5 \mathrm{~kg}$ ). The score for table B is 4 with the upper arm score is 2 (with abducted motion), wrist score is 3, lower arm is 2, and the coupling score is 0 (good). Based on the score result of table $\mathrm{A}$ and table $\mathrm{B}$, thescore for table $\mathrm{C}$ is 8 . So the REBA score is 10 with the activity score is 2 because the task makes her static in standing position more than 1 minutes and the worker does it repetitively. It shows that the rice planting activity has very high risk and needs immediate corrective action because it can lead to MSDs' if done for a long period (Fig.3).

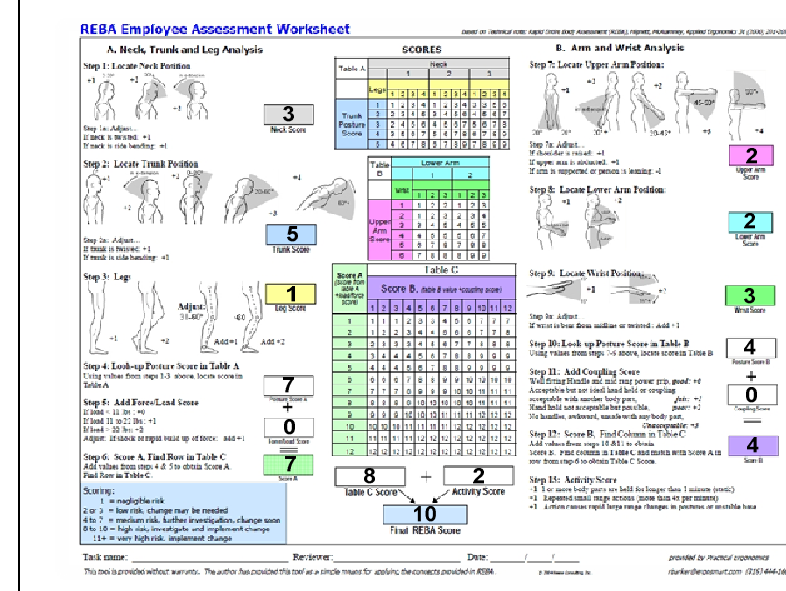

Fig.3 The worksheet for data processing of REBA in existing condition

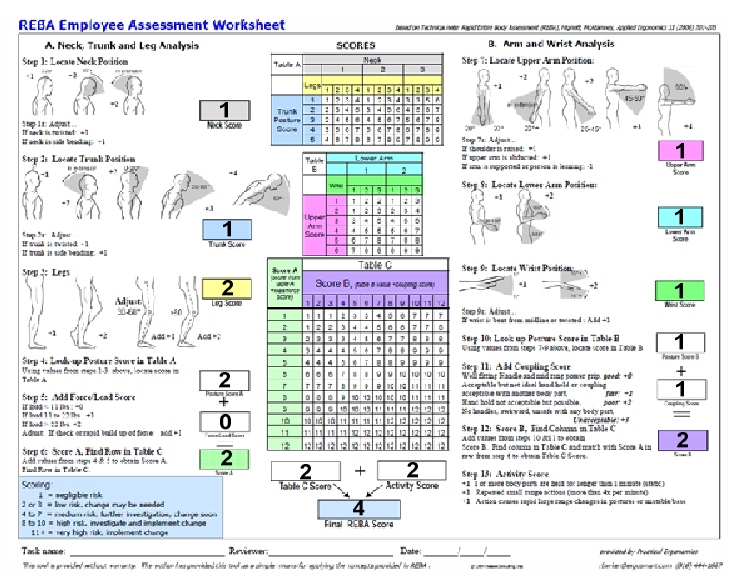

Fig.4 The worksheet for data processing of REBA using new design

After using the proposed rice planter tool, the REBA scoring result in Fig.4 shows thatthe score for table A is 2 with the score for neck is 1 , score for leg is 2 , score for trunk is 1 and the load score is 0 (load $<5 \mathrm{~kg}$ ). The score for table B is 2 with the wrist score is 1 , lower arm is 1 , upper arm score is 1 and the coupling value is 1 (fair). Based on the score result of table A and table B, the score for table $\mathrm{C}$ is 2 . So the REBA score is 4 . It shows that the rice planting activity has medium risk level and needs corrective action.

\section{Anthropometry Data}

Table 1 shows the body dimension measurements that used for designing the rice planter tool based on Anthropometry data:

Table 1: The Measurement of Farmers' Body Dimension

\begin{tabular}{|l|l|c|c|l|c|}
\hline No & \multicolumn{1}{|c|}{ Data Measured } & $\begin{array}{c}\text { Result } \\
{[\mathbf{c m}]}\end{array}$ & No & Data Measured & $\begin{array}{c}\text { Result } \\
{[\mathbf{c m}]}\end{array}$ \\
\hline $\mathbf{1}$ & Standing Elbow Height & 97.75 & $\mathbf{1 0}$ & Thumb Thickness & 1.5 \\
\hline $\mathbf{2}$ & Hand Length & 17.5 & $\mathbf{1 1}$ & Index Finger Breadth & 2 \\
\hline $\mathbf{3}$ & Palm Length & 10 & $\mathbf{1 2}$ & Index Finger Thickness & 1 \\
\hline $\mathbf{4}$ & Thumb Length & 6 & $\mathbf{1 3}$ & Palm Breadth (Metacarpal) & 8 \\
\hline $\mathbf{5}$ & Index Finger Length & 7 & $\mathbf{1 4}$ & Palm Breadth(to Thumb) & 9 \\
\hline $\mathbf{6}$ & Middle Finger Length & 7 & $\mathbf{1 5}$ & Palm Thickness (Metacarpal) & 2 \\
\hline $\mathbf{7}$ & Ring Finger Length & 7 & $\mathbf{1 6}$ & Palm Thickness (to Thumb) & 2.5 \\
\hline $\mathbf{8}$ & Little Finger Length & 5 & $\mathbf{1 7}$ & Palm Maximum Breadth & 2.5 \\
\hline $\mathbf{9}$ & Thumb Breadth & 2 & & & \\
\hline
\end{tabular}

These body dimension values will be used as a measurement of the rice planter tool design, which are for the handle, tool's height and the tool's reachdesign. 


\section{Design of Rice Planter Tool}

Based on the Participatory Ergonomic, NBMQ, REBA scoring also Anthropometry data, theproposed design of rice planter tool as shown in the Fig.5.

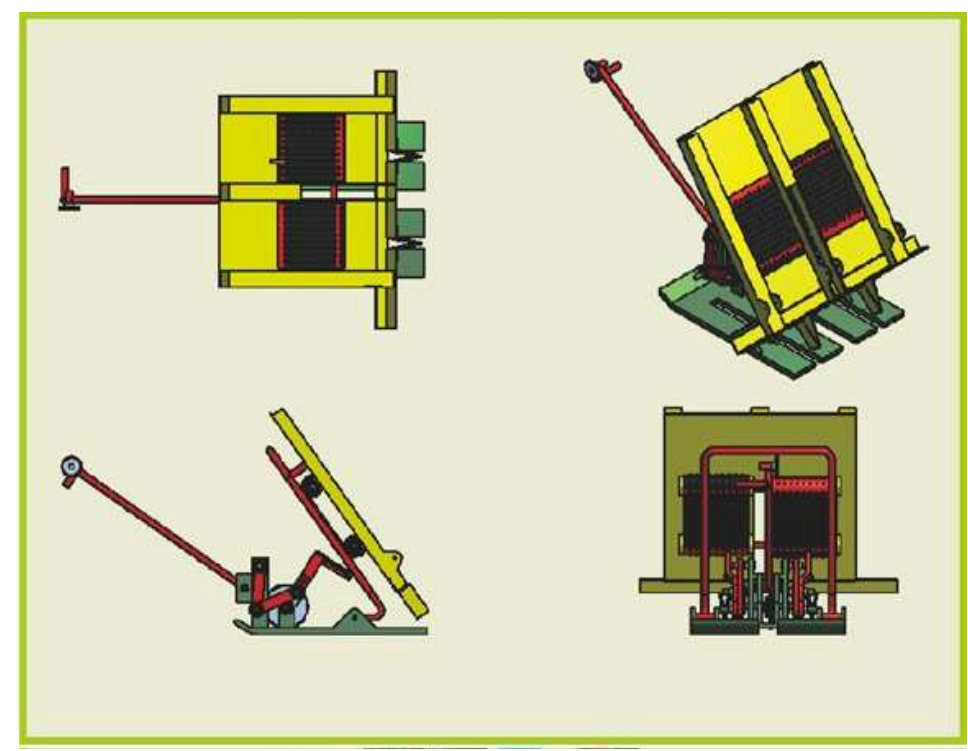

Fig. 5 Proposed Design of Rice Planter Tool

In this tool, there is an activator system to move both of machine's arms. These arms are used to pick the rice and plant it in the field. This tool is used in standing upright condition and backward direction. To use this tool, the rice sprout that can be planted is put in the front part of this tool. The planting process is conducted by the machine's arms which look like human arms. For one time the operator spin the handle of this tool, the both arms will be moved simultaneously to pick the rice from its place to be planted. Thus, principally in one rotation, this tool is able to plant 2 rice sprouts. Suppose the same worker which usually plants one rice sprout with conventional method spends time about $x$ seconds. Compared with using the proposed rice planter tool, the worker only spends $0,5 \mathrm{x}$ seconds from conventional method rice planting. Therefore, the time for rice planting will be more efficient by using this tool.

\section{Discussion}

Based on the result of NBMQ and REBA, it is obtained thatthe work posture during rice planting has the risk of musculoskeletal disorders. The highest injury risk can be seenbased on the REBA scoring result on the trunk is 5, with total score 10 . This result includes in very high risk level and needs immediate corrective action [6]. Therefore, in this research rice planter tool is proposed to reduce the REBA scoring result, specifically on trunk. Accoding to Fig.4, by using this tool, it will reduce the REBA score on the trunk by $(-4)$ and neck $(-2)$. The total score of REBA after using this rice planter tool become 4 (medium risk level). This research also provides a comparison of rice planting using conventional method and the proposed tool in terms of work method, time efficiency and expended cost.

Participatory ergonomic (PE) implementation ensures that those who were directly affected by ergonomic hazards (workers) were actively involved in the identification, assessment and control of these hazards [7]. The Participatory Ergonomic was applied to solve the problem of MSD's in rice planting. Small group involved farmer community in Bantul, tool designer, ergonomic expert, and tool technician took the role in solving the problem. According to the case, the farmers delivered the grievance about MSDs especially in the wrist, back, and shoulder. Then, the ergonomic expert gave the recommendation based on the problem. The tool designer did design the tool that can help the participants in doing planting activity which supported by tool technician who gave recommendation about material and cost of the tool. The proposed design could be seen on the Fig.5. In this case, the author sees that the hazards of the farmers were reduced. 
From the work method side, the rice planter utilization will be able to reduce the risk of musculoskeletal disorders for farmers while planting the rice. This tool is designed in such a manner so the farmers will not in bend position while planting the rice. Bending work posture can be replaced with standing upright posture by spinning the pulley which serves as an activator system of the machine arms to pick and plant the rice.Therefore, the complaints in the back, waist, and shouldersalso will be able to reduced.

Regarding the side of time efficiency, by using the proposed design of rice planter tool, it will minimize the required time to plant the rice in one field because this tool can plants 2 rice sprouts in 1 rotation. If it is compared with the conventional rice planting where the rice sprout only able to be planted one by one, suppose the same worker which usually plants one rice sprout with conventional method spends time about x seconds. Compared with using the proposed rice planter tool, the worker only spends $0,5 \mathrm{x}$ seconds from conventional method rice planting.

\section{Conclusion}

The research can be concluded that the MSDs'complaints on work posture when planting rice using conventional method can be reduced until $66.67 \%$ by using the proposed rice planter tool. In addition, the utilization of this rice planter tool from the work method, time efficiency and expended cost side is better compared with conventional method of rice planting. It is supposed that the same worker which usually plants one rice sprout with conventional method spends time about $\mathrm{x}$ seconds. The time efficiency by using proposed rice planter tool is obtained $0,5 \mathrm{x}$ seconds from conventional method. Therefore, the time for rice planting will be more efficient by using this tool.

\section{References}

[1] Ambedkar, B.R. Work Posture Assessment In Forging Industry: An Exploratory Study in India, Department of Industrial \& Production Engineering, National Institute of Technology Jalandhar., International Journal of Advanced Engineering Technology. 1 (2010) 358-366.

[2] Davis K.G., Understanding The Ergonomic Risk for Musculoskeletal Disorders in The United States Agricultural Sector, Department of Environmental Health, University of Cincinnati, Ohio, USA, 2005.

[3] Karwowski, Waldemar., Salvendy, Gavriel. Ergonomics in Manufacturing, Engineering \& Management Press, Nacros, (1998)

[4] De Jong, AM, A Three-Phased Model of Participatory Ergonomics Processes to Improve Work in The Construction Industry, Industial Health Journal. 30 (2004) 383-387.

[5] Punnett, L., and Wegman, D. H.,Work - Related Musculoskeletal Disorders: The Epidemiologic Evidence and the Debate,J. Electromyography and Kinesiology. 14 (2004) 13 - 23.

[6] Hignett S., and McAtamney L.,Rapid Entire Body Assessment (REBA), Applied Ergonomics. $31(2000) 201-205$.

[7] Morgan D., Participatory Ergonomics: Introducing the OSACH Ergonomic Program Implementation Continuum (EPIC) Program a Practical Approach to Injury Prevention, Ontario Safety Association for Community and Healthcare. 1 (2009) 1-15. 